\title{
SOBREVIDA DE PACIENTES CON CÁNCER GÁSTRICO ETAPAS IIIC Y IV SOMETIDOS A CIRUGÍA. PARTE II DE ESTUDIO PROSPECTIVO 2004-2012*
}

\author{
Drs. Attila Csendes J. ${ }^{1}$, E.U. Solange Cortés L. ${ }^{1}$, \\ Drs. Matias Guajardo B. ${ }^{1}$, Manuel Figueroa G. ${ }^{1}$ \\ 1 Departamento de Cirugía, Hospital Clínico Universidad de Chile. \\ Santiago, Chile.
}

\begin{abstract}
\section{Survival of patients with advanced gastric cancer subjected to palliative surgery}

Background: Most patients with gastric cancer consult when the tumor is an advanced stage. Aim: To assess the survival of patients with gastric cancer in stage IIIc or IV subjected to surgery. Patients and Methods: Prospective study of patients with gastric cancer in stages IIIc (spreading to nearby organs or serosa) or IV (spreading to distant organs), who consulted between 2004 and 2012. A surgical exploration was carried out in all patients performing a palliative total or subtotal gastrectomy, a gastrojejunal anastomosis or only an exploration. Twenty five patients received adjuvant chemotherapy. All were followed until their death. Results: A surgical resection was performed in 61 patients (64\%) with an operative mortality of 5 and $0 \%$ for total and subtotal gastrectomy, respectively. The mortality of non-resected patients was $6.4 \%$ with a maximal survival of 15 months after surgery. No significant differences in survival were observed between patients with hepatic or peritoneal metastases. Adjuvant chemotherapy significantly improved survival among patients subjected to gastric resection. Conclusions: Among patients with gastric cancer, palliative gastrectomy may improve quality of life and adjuvant chemotherapy apparently improves survival.
\end{abstract}

Key words: Gastric cancer, palliative surgery, chemotherapy.

\section{Resumen}

Introducción: El cáncer gástrico constituye un problema de salud pública por su alta incidencia. Muchos pacientes consultan en etapas muy avanzadas. Objetivo: Evaluar la sobrevida de pacientes con cáncer gástrico etapa IIIc y IV sometidos a cirugía. Material y Método: Estudio prospectivo y descriptivo de pacientes en dichas etapas, que consultaron entre mayo de 2004 y diciembre de 2012, divididos en 4 grupos según ubicación de metástasis. Todos sometidos a exploración quirúrgica realizando una gastrectomía total o subtotal paliativa, gastroyeyuno-anastomosis o sólo laparotomía. Quimioterapia adyuvante se realizó en 25 pacientes. Se realizó un seguimiento hasta la fecha de fallecimiento en un $100 \%$ de los casos. Resultados: La

*Recibido el 2 de enero de 2014 y aceptado para publicación el 7 de abril de 2014.

Los autores declaran no tener conflicto de interés.

Correspondencia: Attila Csendes J. acsendes@hcuch.cl 
resección quirúrgica se realizó en 61 pacientes $(64,3 \%)$, con una mortalidad operatoria de 4,6\% para la gastrectomía total y $0 \%$ para la gastrectomía subtotal. La mortalidad de los pacientes no resecados fue de $6,4 \%$, con sobrevida máxima de 15 meses después de operado. Entre los casos con resección gástrica, se observó una mayor pero no significativa sobrevida en pacientes con metástasis hepáticas comparadas con las peritoneales. La quimioterapia adyuvante mostró una sobrevida significativamente mayor en los resecados comparado a los pacientes en los que no se la empleó. Conclusión: La gastrectomía paliativa para mejorar la calidad de vida en pacientes con cáncer gástrico metastásico es factible, en grupos entrenados. El efecto beneficioso de la quimioterapia adyuvante debe ser evaluado en futuros estudios randomizados.

Palabras clave: Cáncer gástrico, metástasis peritoneales, metástasis hepática, invasión a órganos vecinos, quimioterapia adyuvante.

\section{Introducción}

El cáncer gástrico sigue siendo una enfermedad de gran importancia clínica, ya que representa la $1^{\circ}$ causa de mortalidad por tumores malignos, con 3.000 fallecidos anualmente. Su incidencia se ha mantenido estable en la última década con una tasa cercana a 20 pacientes por 100.000 habitantes ${ }^{1}$.

En nuestro país ha habido una importante dedicación a este tema con desarrollo de numerosos equipos quirúrgicos a lo largo de Chile, que han obtenido muy buenos resultados tanto en la disminución de la morbimortalidad operatoria, el aumento de pacientes con cáncer incipiente, el aumento de la resecabilidad y un aumento en la sobrevida ${ }^{2,3}$.

Sin embargo, aún hay un número importante de pacientes, que consultan en una etapa IIIc y IV, es decir, con invasión a serosa o metástasis a distancia. En 1974 publicamos el primer estudio sobre la sobrevida a 5 años de un grupo de 752 pacientes con cáncer gástrico ${ }^{4}$. La sobrevida global de los pacientes resecados fue de $19 \%$ y los no resecados de 0 . En el presente estudio el objetivo fue determinar la sobrevida de pacientes con cáncer gástrico etapa IIIc y IV, incluidos en un seguimiento prospectivo, para determinar si la resección quirúrgica en esta etapa logra un aumento de la misma.

\section{Material y Método}

\section{Pacientes estudiados}

El presente estudio es un resultado de un protocolo prospectivo de seguimiento de todos los pacientes con tumores malignos que se atienden en el Departamento de Cirugía del Hospital Clínico de la Universidad de Chile, a partir de mayo de 2004, cuando se creó una Unidad de Oncología a cargo de una E. U. especializada en esta patología (S.C.). Todos los pacientes son ingresados a una planilla Excel durante su hospitalización, incluyendo todas las variables clínicas, de laboratorio, anátomo-patológicas, quirúrgicas y evolución postoperatoria ${ }^{5}$. Se realiza un seguimiento comprobando el estado actual o la fecha exacta del fallecimiento del paciente.
Por lo tanto se incluyeron a todos los pacientes con Adenocarcinoma gástrico que se hospitalizaron en el período mayo de 2004 a diciembre de 2012. Se excluyeron pacientes con linfoma gástrico, GIST maligno o cáncer del muñón gástrico. Para el presente estudio se incluyeron solamente los pacientes en etapas IIIc y IV, según la última edición de la clasificación del cáncer gástrico del $2010^{6-8}$, En esta última edición se define estas etapas avanzadas de la siguiente manera.

a) Estadio III $\begin{array}{llll}\mathrm{T}_{4 \mathrm{~b}} & \mathrm{~N}_{2} \circ \mathrm{N}_{3} & \mathrm{M}_{0} \\ & \mathrm{~T}_{4 \mathrm{a}} & \mathrm{N}_{3} & \mathrm{M}_{0}\end{array}$

b) Estadio IV cualquier T cualquier $\mathrm{N} \quad \mathrm{M}_{1}$

$\mathrm{T}_{4 \mathrm{a}}$ : Corresponde a invasión de la serosa o peritoneo visceral.

$\mathrm{T}_{4 \mathrm{~b}}$ : Invasión de estructuras vecinas como bazo, páncreas, colon transverso, hígado, diafragma riñón, glándula adrenal.

$\mathrm{N}_{2}$ : Metástasis de 3 a 6 linfonodos.

$\mathrm{N}_{3}^{2}$ : Metástasis de 7 o más linfonodos.

$M_{1}^{3}$ : Metástasis a distancia, que incluye citología peritonal positiva. En el presente estudio, se excluyeron los pacientes T4a, es decir, con invasión de la serosa exclusivamente.

Los pacientes con metástasis hepáticas todos tenían entre 1 y 5 metástasis, pero sin otro compromiso a distancia.

Los pacientes con metástasis peritoneales incluidos en este estudio correspondieron a aquellos con finas metástasis tanto en el espacio supra como infra cólico, pero no en cantidad muy extensa y todos con ausencia de ascitis. Los pacientes con metástasis mixtas tenían tanto escasas metástasis hepáticas como peritoneales.

El grupo de compromiso a otros órganos correspondió a infiltración de órganos vecinos como colon, páncreas, diafragma, hígado o suprarrenales.

\section{Técnica quirúrgica}

Todos los pacientes incluidos en esta investigación fueron sometidos a cirugía paliativa, con el objeto de mejorar la calidad de vida con una resección gástrica paliativa. 
Sin embargo, en algunos se realizó solamente una laparotomía exploradora, o una derivación tipo gastroyeyuno-anastomosis. La resección gástrica fue total o subtotal, con una reconstitución del tránsito intestinal con un asa en Y-de-Roux de $70 \mathrm{~cm}$ de largo. No se realizó disección linfática D2 reglada en ningún paciente por tener un cáncer avanzado, que no justificaba una cirugía radical.

\section{Estudio histológico}

Para la clasificación del tipo histológico del cáncer gástrico, se empleó la nomenclatura propuesta por Lauren', quien lo divide en tipo intestinal o difuso.

\section{Seguimiento}

Se efectuó un seguimiento clínico en el $100 \%$ de los pacientes, determinando a marzo de 2013 si estaban vivos o el momento de su fallecimiento, tanto en los registros clínicos como lo informado por el Servicio de Registro Civil.

\section{Quimioterapia}

Este tratamiento se realizó en 25 pacientes después de la cirugía y no se realizó en 64 pacientes. Esto da un total de 89 pacientes, ya que 4 fallecieron en el postoperatorio. Las drogas empleadas fueron: 5 fluoruracilo, etopósido y cisplatino, realizando en promedio 3 ciclos de quimioterapia.

\section{Cálculos estadísticos}

Se empleó el promedio \pm desviación estándar para algunos cálculos. La sobrevida se expresó de acuerdo a los cálculos estadísticos de sobrevida actuarial según el método de Kaplan-Meier.

La significación estadística se calculó mediante la prueba de $\chi^{2}$, Test de Log-Rank (Mantel-Cox), Test de Wilconxon y Mantel-Haenszel tomando un $\mathrm{p}<0,05$ como significativo.

\section{Resultados}

En el período de estudio (mayo de 2004-diciembre de 2012) se atendieron un total de 430 pacientes con Adenocarcinoma gástrico. De ellos, 92 pacientes, es decir, un $21,4 \%$ estaba en etapa IIIc, IV, excluyendo a los pacientes con T4a.

Los pacientes se clasificaron en 4 grupos, de acuerdo a la extensión del tumor:

a) Grupo I: metástasis peritoneales, que correspondieron a 57 pacientes $(61,9 \%)$.

b) Grupo II: metástasis hepáticas, que fueron 11 pacientes $(11,9 \%)$.

c) Grupo III: metástasis mixtas, tanto peritoneales como hepáticas que fueron 10 pacientes $(10,8 \%)$.

d) Grupo IV: invasión a otros órganos $=14$ pacientes $(15,2 \%)$.

En la Tabla 1 se muestra las principales características epidemiológicas, con edades promedio similares en los 4 grupos. Hubo un predominio de 1,5 hombres por 1 mujer. La duración de los síntomas fue similar en los 4 grupos. La Tabla 2 señala los principales síntomas en cada grupo. La baja de peso, el dolor abdominal y vómitos fueron los síntomas más frecuentes en los 4 grupos. No hubo diferencia significativa en la proporción de síntomas en cada grupo.

Las operaciones realizadas en este grupo de pacientes se observan en la Tabla 3. Hubo 31 pacientes $(35,7 \%)$ en los que no se pudo realizar una resección gástrica por lo avanzado de la enfermedad. Sin embargo, en 61 pacientes $(64,3 \%)$ se pudo efectuar una gastrectomía total o subtotal de tipo paliativa. También se señala la mortalidad operatoria de las diferentes operaciones. Hubo 2 fallecidos después de gastrectomía total: 1 en el grupo de metástasis peritoneales por filtración de la anastomosis esófago yeyunal y falla orgánica múltiple. Otro paciente del grupo mixto falleció por sepsis de foco pulmonar, sin complicación abdominal. Hubo 2 fallecidos en el

Tabla 1. Cirugía en pacientes con cáncer gástrico etapa IIIc y IV (2004-2012). $\mathrm{N}=92$

\begin{tabular}{|c|c|c|c|c|c|}
\hline & \multicolumn{3}{|c|}{ Compromiso tumoral } & \multirow{2}{*}{$\begin{array}{c}\text { Invasión otros } \\
\text { órganos } \\
\mathrm{n}=14\end{array}$} & \multirow[t]{2}{*}{$\mathbf{p}$} \\
\hline & $\begin{array}{c}\text { Peritoneales } \\
\mathbf{n}=\mathbf{5 7}\end{array}$ & $\begin{array}{c}\text { Hepáticas } \\
\mathbf{n}=\mathbf{1 1}\end{array}$ & $\begin{array}{c}\text { Mixtas } \\
n=10\end{array}$ & & \\
\hline Edad x (años) & $57,6 \pm 10,5$ & $60,1 \pm 12,6$ & $56,9 \pm 9,8$ & $65,5 \pm 12,3$ & NS \\
\hline $\begin{array}{l}\text { Género } \\
\text { Hombres } \\
\text { Mujeres }\end{array}$ & $\begin{array}{c}33(57,9 \%) \\
24\end{array}$ & $\begin{array}{c}9(81,8 \%) \\
2\end{array}$ & $\begin{array}{c}5(50 \%) \\
5\end{array}$ & $\begin{array}{c}10(71 \%) \\
4\end{array}$ & NS \\
\hline Duración síntomas (meses) & $5 \pm 5,3$ & $4,8 \pm 4,3$ & $10,1 \pm 15$ & $3,2 \pm 7,8$ & NS \\
\hline
\end{tabular}

$\mathrm{NS}=$ no significativo. 
Tabla 2. Síntomas de pacientes con cáncer gástrico etapas IIIc y IV sometidos a cirugía. $\mathrm{N}=92$

\begin{tabular}{|c|c|c|c|c|c|}
\hline \multirow[t]{2}{*}{ Tipo síntomas principales (\%) } & \multicolumn{3}{|c|}{ Compromiso tumoral } & \multirow{2}{*}{$\begin{array}{c}\text { Invasión otros } \\
\text { órganos } \\
n=14\end{array}$} & \multirow[t]{2}{*}{$\mathbf{p}$} \\
\hline & $\begin{array}{c}\text { Peritoneales } \\
\mathbf{n}=\mathbf{5 7}\end{array}$ & $\begin{array}{c}\text { Hepáticas } \\
\mathbf{n}=11\end{array}$ & $\begin{array}{c}\text { Mixtas } \\
\mathbf{n}=10\end{array}$ & & \\
\hline Baja de peso & 67,9 & 72,7 & 70,0 & 60 & NS \\
\hline Dolor & 54,7 & 54,5 & 80,0 & 66,7 & NS \\
\hline Vómitos & 32,1 & 18,2 & 20,0 & 33,3 & NS \\
\hline Saciedad precoz & 18,9 & 9,1 & 10,0 & 20,0 & NS \\
\hline Anemia crónica & 13,2 & 9,1 & 40,0 & 13,3 & NS \\
\hline Disfagia & 20,8 & 18,2 & 20,0 & 13,3 & NS \\
\hline
\end{tabular}

$\mathrm{NS}=$ no significativo.

Tabla 3. Operaciones efectuadas y mortalidad operatoria en pacientes con cáncer gástrico etapa IIIc y IV. $\mathrm{N}=92$

\begin{tabular}{|lccccc|}
\hline Operación principal & \multicolumn{3}{c}{ Compromiso tumoral } & Peritoneales \\
$\mathbf{n}=\mathbf{5 7}$ & Hepáticas & $\mathbf{n = 1 1}$ & $\begin{array}{c}\text { Mixtas } \\
\mathbf{n}=\mathbf{1 0}\end{array}$ & $\begin{array}{c}\text { Invasión otros } \\
\text { órganos } \\
\mathbf{n}=\mathbf{1 4}\end{array}$ & $\begin{array}{c}\text { Mortalidad } \\
\text { operatoria }\end{array}$ \\
Gastrectomía total & 27 & 6 & 4 & 6 & $2 / 43=4,6 \%$ \\
Gastrectomía subtotal & 10 & 2 & 2 & 4 & $0 / 18=0$ \\
Lap. Exploradora & 20 & 1 & 4 & 0 & $2 / 25=8 \%$ \\
Gastroyeyuno-Anastomosis & 0 & 2 & 0 & 4 & $0 / 6=0$ \\
Fallecidos postop & 3 & 0 & 1 & 0 & $4 / 92=4,3 \%$ \\
\hline
\end{tabular}

Tabla 4. Histología de pacientes con cáncer gástrico etapas IIIb y IV sometidos a cirugía

\begin{tabular}{|lccc|}
\hline \multicolumn{4}{|c|}{ Clasificación de Lauren } \\
Tipo de metástasis & Intestinal & Difuso & $\begin{array}{c}\text { Proporción } \\
\text { I/D }\end{array}$ \\
$\begin{array}{l}\text { Peritoneales } \\
\mathrm{n}=57\end{array}$ & 19 & 34 & $1: 2$ \\
$\begin{array}{l}\text { Hepáticas } \\
\mathrm{n}=11\end{array}$ & $(35,8 \%)$ & $(64,2 \%)$ & \\
$\begin{array}{l}\text { Mixtas } \\
\mathrm{n}=10\end{array}$ & $\begin{array}{c}8 \\
(72,7 \%)\end{array}$ & $(27,3 \%)$ & $3: 1$ \\
$\begin{array}{l}\text { Invasión a otros órganos } \\
\mathrm{n}=14\end{array}$ & $5(50 \%)$ & $5(50 \%)$ & $1: 1$ \\
\hline I/D $=$ intestinal-difuso. & $94 \%)$ & $5(36 \%)$ & $1.5: 1$ \\
\hline
\end{tabular}

grupo de laparotomía exploradora por sepsis y falla orgánica múltiple.

En la Tabla 4 se detallan los tipos histológicos de cáncer gástrico según la clasificación de Lauren. Se aprecia que en pacientes con metástasis peritoneales predomina el tipo difuso, mientras que en pacientes con metástasis hepáticas predomina el tipo intestinal. No hubo diferencia significativa comparando los grupos entre sí.

La sobrevida de todos los pacientes según el tipo de metástasis se muestra en la Figura 1. Se aprecia que a los 20 meses de operados casi el $90 \%$ de los pacientes ha fallecido sin diferencia significativa comparando la sobrevida $(\mathrm{p}>0,24)$.

La sobrevida actuarial de cada grupo de pacientes según si se efectuó o no una resección quirúrgica paliativa se muestra en las Figuras 2, 3, 4 y 5. En la Figura 2 se aprecia que la sobrevida en pacientes con metástasis peritoneales fue significativamente mayor $(\mathrm{p}<0,003)$ con resección gástrica comparado con la no resección. Lo mismo ocurre en pacientes con metástasis hepáticas $(\mathrm{p}<0,02)$ y metástasis mixtas $(\mathrm{p}<0,002)$. En pacientes con infiltración a otros órganos no hubo diferencia significativa ( $p>0,17)$. La sobrevida media, que es el momento de fallecimiento del $50 \%$ de los casos se expresa en la Tabla 5, expresando los mismos resultados que en las figuras ya mencionadas.

Al comparar la sobrevida después de gastrectomía total o subtotal no se apreció diferencia significativa en cada uno de los grupos estudiados. 

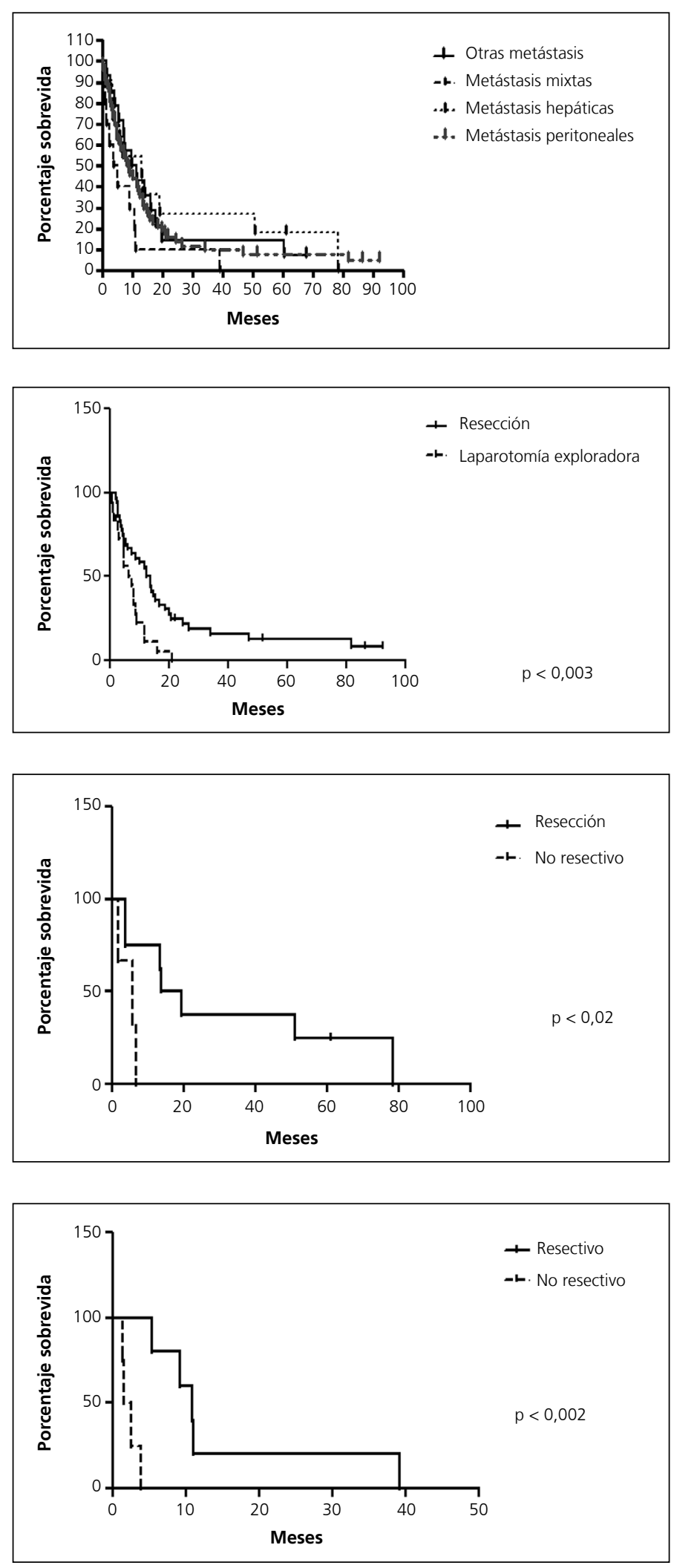

Figura 1. Sobrevida de pacientes con cáncer gástrico etapa IIIc y IV según tipo de metástasis a distancia.

Figura 2. Sobrevida de pacientes con metástasis peritoneales sometidos a resección o no resección gástrica.

Figura 3. Sobrevida de pacientes con metástasis hepáticas sometidos a resección o no resección gástrica.

Figura 4. Sobrevida de pacientes con metástasis mixtas sometidos a resección o no resección gástrica. 
En la Figura 6 se muestra la sobrevida de pacientes con metástasis peritoneales sometidos a gastrectomía total o subtotal. Hubo 15 pacientes con quimioterapia adyuvante y 38 en lo que no se empleó. La sobrevida fue significativamente mayor $(p<0,001)$ en los pacientes con quimioterapia. El mismo efecto se muestra en la Figura 7, en la que hubo 4 pacientes con metástasis hepáticas sometidos a quimioterapia y 8 sin este tratamiento. La sobrevida en pacientes resecados fue significativamente mayor con quimioterapia $(\mathrm{p}<0,003)$. En los otros 2 grupos no se observó diferencias significativas, así como en ningún grupo en los que sólo se realizó una laparotomía exploradora.
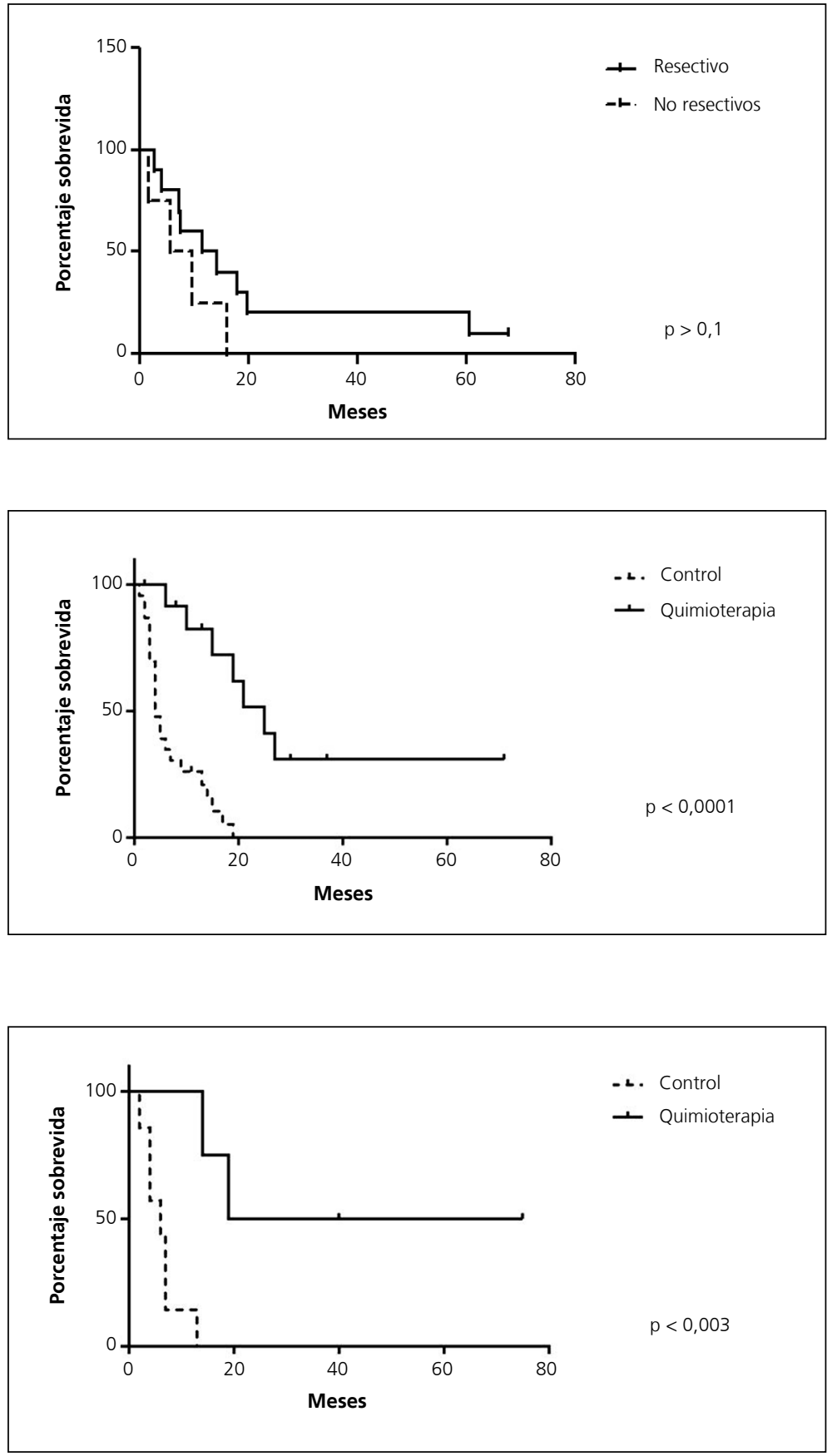

Figura 5. Sobrevida de pacientes con invasión a otros órganos sometidos a resección o no resección gástrica.
Figura 6. Meta peritoneal: gastrectomía total + GST: QMT vs no QMT.
Figura 7. Meta hepática: gastrectomía total + GST: QMT vs no QMT. 
Tabla 5.

\begin{tabular}{|lcc|}
\hline Sobrevida media (meses) & Laparotomía & Resección \\
Metástasis peritoneales & 7,2 & 12,9 \\
Metástasis hepáticas & 5,8 & 16,3 \\
Metástasis mixtas & 2,0 & 10,9 \\
Otros órganos & 7,6 & 12,8 \\
\hline
\end{tabular}

\section{Discusión}

Los resultados del presente estudio prospectivo sugieren en primer lugar que en pacientes con cáncer gástrico muy avanzado, etapa IIIc y IV, la resección quirúrgica puede ofrecer una sobrevida algo mayor comparada con realizar sólo una laparotomía exploradora y probablemente una mejor calidad de vida, lo que no fue evaluado en este estudio. En segundo lugar los resultados de esta cirugía paliativa son mucho mejores que los reportados por nosotros hace 40 años ${ }^{4}$.

Al revisar la literatura nacional de las últimas décadas, llama la atención las escasas publicaciones referentes a estos pacientes en etapas terminales. Casi todos los reportes se refieren a cirugías resectivas en etapas más precoces de la enfermedad. Bettina y cols ${ }^{10}$, relatan que un $38 \%$ de los pacientes con cáncer gástrico están en etapa IV, que es mayor a nuestra cifra de $22 \%$, probablemente por ser nosotros un centro de referencia, consultando pacientes más seleccionados. García y cols ${ }^{11}$, mencionan una sobrevida de $3,4 \%$ en cirugía no curativa, cifra muy menor comparado a nuestros resultados.

En los últimos 40 años ha habido notables avances en el diagnóstico y tratamiento de pacientes con cáncer gástrico ${ }^{12}$ : la resecabilidad ha aumentado de un $40 \%$ a sobre $80 \%$, la mortalidad operatoria ha bajado de $28 \%$ a $2 \%{ }^{13}$, la proporción de pacientes con cáncer incipiente ha aumentado y la sobrevida ha mejorado notablemente. En 1974 describíamos una vida media de 12 meses para pacientes con resección gástrica, con una sobrevida de $19 \%$ a 5 años versus 2 meses de vida media para los no resecados ${ }^{4}$.

En el presente estudio hemos querido profundizar algo más en cuanto al tipo de paciente, tipo de operación y sobrevida en casos con cáncer muy avanzado. Hemos subdividido a estos pacientes en 4 grupos principales, de acuerdo a la extensión del tumor. Pacientes sometidos sólo a exploración quirúrgica tienen la misma sobrevida que hace 40 años y no hay ninguna mejoría. Por el contrario, pacientes en los que se puede realizar una resección gástrica total o subtotal aún en presencia de metástasis, muestran una mayor sobrevida, que es lo destacable. Com- parando la sobrevida de los 4 grupos de pacientes llama la atención que: a) se aprecia mayor pero no significativa sobrevida en pacientes con metástasis hepáticas solamente, comparados con los otros grupos y b) una mejor sobrevida en pacientes sometidos a gastrectomía total o subtotal que fue significativa. Este hecho ha sido publicado por varios autores extranjeros ${ }^{14-26}$. Se ha sugerido que el agregado de quimioterapia postoperatoria en pacientes con resección paliativa parece estar asociada a una mayor sobrevida que con la cirugía sola ${ }^{19,22,23}$.

En nuestro estudio los resultados de los pacientes sometidos a resección gástrica más quimioterapia adyuvante muestran una diferencia significativamente mayor en la sobrevida comparado con pacientes resecados sin quimioterapia posterior, pero sólo en los grupos con metástasis peritoneales o con metástasis hepáticas. Por el contrario en pacientes con metástasis mixta o con invasión a otros órganos, así como en pacientes sometidos sólo a una laparotomía, no se apreció un efecto beneficioso de la quimioterapia.

Para realizar una gastrectomía total o subtotal de tipo paliativo en pacientes con metástasis a distancia se deben cumplir algunos requisitos: por parte del paciente se requiere estar en condiciones clínicas y anestésicas compatibles con la cirugía, lo que significa una juiciosa selección del paciente ${ }^{21,25}$. Por parte del equipo quirúrgico se requiere una amplia experiencia en cirugía gástrica, que le ofrezca al paciente una baja morbimortalidad. En el presente estudio nuestra mortalidad operatoria de gastrectomía total fue $4,6 \%$ y de la subtotal $0 \%$, cifras que nos parecen adecuadas para la complejidad de la cirugía. Autores extranjeros mencionan cifras de mortalidad operatoria de $6 \%$ a $8,7 \%{ }^{25-27}$.

En el presente estudio no hemos evaluado la calidad de vida después de gastrectomía paliativa y eventual quimioterapia. La OMS señala claramente que el alivio de síntomas debe ser el foco primario del tratamiento paliativo. La revisión Cochrane ${ }^{17}$ mostró 9 artículos referentes a este tema, en el período postoperatorio inmediato, pero ninguno reportó estudios de calidad de vida alejada, usando los instrumentos validados para este objetivo.

La fortaleza del presente estudio está por una parte que es prospectivo, siguiendo un protocolo establecido el año 2004. Por otra parte, el equipo quirúrgico tiene una amplia experiencia en este tema, lo que representa una mayor seguridad para los pacientes. Este hecho permitió realizar cirugías resectivas en pacientes en quienes décadas atrás no se hubiera realizado una resección gástrica.

La debilidad de este estudio reside en el hecho que los pacientes sometidos a laparotomía o resección quirúrgica no son randomizados. Por el 
contrario, son grupos seleccionados, ya que los casos con metástasis hepáticas o peritoneales que no se resecaron tenían un mucho mayor compromiso clínico que los resecados, por lo que los resultados están influidos por la extensión de la enfermedad. Otra debilidad de este estudio fue el hecho que el tratamiento con quimioterapia adyuvante no fue randomizado y sólo se realizó en un pequeño grupo de pacientes ( 25 de 89 casos). Por esto, las conclusiones son sólo sugerencias, pero no pueden tener una fuerte validez científica.

En conclusión, en pacientes con cáncer gástrico avanzado etapa IIIc se puede realizar la cirugía completa R0 tipo D2, lo que no se puede lograr en una etapa IV. Sin embargo, la cirugía paliativa parece tener un mejor resultado comparada con décadas anteriores, tanto por mejor evaluación, preparación y por cirugía menos agresiva. El desafío actual es definir qué pacientes se beneficiarán de cirugía más agresiva en estas etapas terminales. Encontrando el adecuado balance entre la agresividad necesaria para extender la sobrevida al mismo tiempo que ofreciendo una mejor calidad de vida es la decisión que debe considerar el equipo quirúrgico.

\section{Referencias}

1. Rajs D. Estadísticas de mortalidad por tumores malignos, MINSAL 2010.

2. Csendes A, Smok G, Medina E. Características evolutivas del cáncer gástrico 1958-1990. Rev Med Chile 1992;120:36-2.

3. Csendes A, Braghetto I, Díaz JC, Castillo J, Rojas J, Cortés S. Morbilidad y mortalidad operatoria de la gastrectomía subtotal y total por cáncer gástrico. Parte I de un estudio prospectivo. Rev Chil Cir. 2011;63:585-90.

4. Csendes A, Medina E. Sobrevida de pacientes con cáncer gástrico I. Sobrevida global en casos operables y no operables. Rev Med Chile 1974;102:837-40.

5. Cortés S, Csendes A, Yarmuch J. Resultados de la Unidad de Oncología del Departamento de Cirugía en un Hospital Universitario (2004-2010). Rev Chil Cir. 2011;63:534-37.

6. Washington K. 7th Edition of the AJCC Cancer Staging Manual Stomach. Ann Surg. Oncol. 2010;17:3077-79.

7. Rodríguez JM, Sasako M, Osorio J. TNM 7a Edición 2009 (UICC/AJCC) y clasificación japonesa 2010 en cáncer gástrico. Hacia la simplicidad y estandarización en el manejo del cáncer gástrico. Cir Esp. 2011;89:27581.

8. Yoon HM, Raju KW, Nama BH, Cho SJ. Is the new seventh AJCC/UICC staging system appropriate for patients with gastric cancer? J Am Coll Surg. 2012;214:88-6.

9. Lauren P. The two histological main types of gastric carcinoma: diffuse and so-called intestinal type carcinoma. Acta Pathol Microbiol Scand. 1965;64:31-49.

10. Bettina M, De La Fuente H, Barajas O, Cardemil B, Mordorovich A. Registro de evaluación del tratamiento de cáncer gástrico en Chile (REGATE). Características clínicas basales de 523 pacientes. Rev Chil Cir. 2011;63:147-53.

11. García C, Benavides C, Apablaza S, Rubilar P, Covacevich $\mathrm{S}$, Peñaloza $\mathrm{P}$, y cols. Resultados del tratamiento quirúrgico del cáncer gástrico. Análisis de 423 casos. Rev Med Chile 2007;135:687-95.

12. Csendes A, Burdiles P, Braghetto I, Díaz JC, Maluenda F, Korn O, y cols. Resecabilidad y mortalidad operatoria de la gastrectomía subtotal y total en pacientes con cáncer gástrico avanzado entre 1969 y 2004. Rev Med Chile 2006;134:426-32.

13. Csendes A, Medina E. Sobrevida de pacientes con cáncer gástrico. III sobrevida del cáncer avanzado según tipo de resección quirúrgica. Rev Med Chile 1975;103:244-46.

14. Li Ch, Yan M, Zhu ZG. Non palliative surgical resection for gastric cáncer patients with distant metástasis. J Invest Surg. 2012;25:100-06.

15. Hundahl SA. Surgery for gastric cáncer: What the trials indicate. Surg Oncol N Am. 2012;79-97.

16. Shindrara S, Korenaga D, Edagawa A, Koushi K, Itali S. Significant prognostic factors in patients with stage IV gastric cáncer with special reference to the curability of surgery. Surg Today 2013;43:40-7.

17. Mahar A, Coburn N, Karanicolas P, Viola R, Helyer L. Effective palliation and quality of life outcomes in studies of surgery for advanced, non curative gastric cancer: a systematic review. Gastric Cancer 2012;15:13845.

18. Kulig P, Sierzega M, Kowalezyk T, Kolodziejczyk P, Kulig J. Non curative gastrectomy for metastatic gastric cancer: Rationale and long-term outcome in multicenter settings. EJSO 2012;38:490-96.

19. Sougioultzis S, Syrios J, Xynos D, Bovaretos N, Kosmas C, Sarantonis J, et al. Palliative gastrectomy and other factors affecting overall survival in stage IV gastric adenocarcinoma patients receiving chemotherapy: A retrospective analysis. EJSO 2011;37:312-18.

20. Saito H, Yamada Y, Tsujitani S. Clinico pathologic characteristics of gastric cancer patients who underwent noncurative gastrectomy with long term survival. Arch Surg. 2009;394:99-03.

21. Dittmar Y, Rauchfuss F, Goetz M, Jandt K, Scheuerlein $\mathrm{H}$, Helse $\mathrm{M}$, et al. Non-curative gastric resection for patients with stage 4 gastric cancer-a single center experience and current review of literature. Arch Surg. 2012;397:745-53.

22. Karpech Jr M. Palliative treatment and the role of surgical resection in gastric cancer. Dig Surg. 2013:30:17480.

23. Yano M, Shiozaki H, Inoue M, Tamura S, Doki Y, 
Yasuda T, et al. Neoadjuvant chemotherapy followed by salvage surgery: Effect on survival of patients with primary noncurative gastric cancer. World J Surg. 2002;26:1155-59.

24. Hung-Hung $\mathrm{K}$, Wun $\mathrm{Wu} \mathrm{C}$, Liang-Fang $\mathrm{W}$, Hao-Chen J, Shun Lo S, Fang-Wang R, et al. Palliative resection in noncurative gastric cancer patients. World J Surg. 2010;34:1015-21.
25. Miner T, Jaques D, Karpeh M, Brennan M. Defining palliative surgery in patients receiving non curative resections for gastric cancer. J Am Coll Surg. 2004;198:1013-21.

26. Schmidt B, Look-Hong N, Maduekwe U, Chang K, Hong T, Kwak E, et al. Non curative gastrectomy for gastric adenocarcinoma should only be performed in highly selected patients. Ann Surg. 2013;20:3512-18. 